\title{
A Study on Room-Level Accuracy of Wi-Fi Fingerprinting-Based Indoor Localization Systems
}

\author{
Umut Can Çabuk ${ }^{1 *}$, Feriştah Dalkılıç ${ }^{2}$, Orhan Dağdeviren ${ }^{3}$ \\ ${ }^{1}$ Department of Electrical and Electronics Engineering, Erzincan University, Erzincan, Turkey \\ ${ }^{2}$ Department of Computer Engineering, Dokuz Eylul University, Izmir, Turkey \\ ${ }^{3}$ International Computer Institute, Ege University, Izmir, Turkey \\ *ucabuk@erzincan.edu.tr
}

Received: 19 April 2018

Accepted: 22 January 2019

DOI: $10.18466 /$ cbayarfbe. 416970

\begin{abstract}
Global positioning system and other outdoor positioning mechanisms are already subject to comprehensive research and development for almost half a century. Conversely, indoor positioning services became a hot topic in the last decade. Since GPS (and. other outdoor solutions) do not work reliably in most indoor environments, researchers and developers are working on accurate positioning solutions, especially tailored for indoor places. However; due to walls, furniture, people and other obstacles, absolute location estimation is very hard and expensive to achieve in indoor places. In addition, accuracy needs depend on the scenario and application. In this study, we have studied the feasibility of room-level location detection in home and office environments. We have focused on examining the quality of room-wise detection accuracy of the fingerprinting method that is applied along with standard $\mathrm{Wi}-\mathrm{Fi}$ radio infrastructure. We have conducted experiments in a multi-storey office building made of concrete and aerated concrete bricks with many rooms, in which it is significantly hard to accurately estimate the correct place of a thing, using radio signals. To the best of our knowledge, our paper is the first study that investigates the room-level accuracy of WiFi fingerprinting-based indoor localization systems. We have found out that, it is possible to feasibly achieve room-level detection with good accuracy, via a pre-calculated room-specific received signal strength indicator threshold value.
\end{abstract}

Keywords: Indoor Positioning, Localization, Wi-Fi, Fingerprinting, Received Signal Strength Indicator.

\section{Introduction}

Right after the big boom of the Global Positioning System (GPS) and its widespread applications in the first decade of the 21st century, Indoor Positioning Systems (IPS) was appeared as a new demand. Hence, researchers and developers started to study on efficient ways to estimate a device's or a person's (in fact any creature's) position indoors. An IPS usually relies on the following three main methodologies regardless of the preferred wireless network technology. These methodologies are proximity, fingerprinting and multilateration [1]. While all these methodologies utilize radio signals originating from external pre-known sources (called as anchor nodes, access points, beacons etc.), their handling of these signals are different.

The proximity methodology simply implies tracking devices' positions via many fixed anchor nodes. Here, a device's position is assumed as the same as the position of the anchor node, which is the source of the strongest received signal (usually the nearest anchor), if there are more than one anchor in the range. In fact, in many applications related to tracking of production lines and logistic processes, there are usually only one anchor in the range at a time. Radio frequency identification (RFID) tags and readers, optic scanners, infrareds, lasers, and ultrasonic devices are usually used for these scenarios.

Nevertheless, fingerprinting is a more complicated method, in which the field-of-interest is virtually divided into grid-like sectors. Before the deployment phase, for each sector, a fingerprint measurement must be made. So that a database shall be built, in order to keep all sectors' signal data. This data is usually in the form of received signal strength indicator (RSSI) values for all access points (AP) in the range of the subject device. This fingerprinting process should be repeated for each sector to complete the database. After the deployment, all devices in the field (which have the full database installed beforehand), can compare their instant RSSI values with the ones in the database and then can decide its own location in the field. These databases can also be stored online, where applicable.

Multilateration, on the other hand, is the generalized version of trilateration, which implies calculation of geometrical (triangular) distances for the subject device 
to determine its position per to access points'. Here, it is assumed that the positions of the access points (anchors) are already known, and the distance factor is precalculated as a function of the RSSI of these access points. In multilateration, unlike fingerprinting, distances are exhaustively computed in real-time without using a pre-defined database, which makes such systems less energy-efficient.

While there is no best solution for all possible use cases, each application scenario shall require a comprehensive decision regarding the correct choice [2]. In this work, we have chosen the fingerprinting approach. Mainly because, we want to achieve (and test) room-level positioning accuracy, where the relative location (localization) is much more important than the absolute distance to the anchor points. Thus, fingerprinting provides advantages in terms of ease of use (and deployment). Moreover, Wi-Fi is considered because of its widespread existence at present, in many different environments, such as shopping malls, schools, hospitals, offices etc. To the best of our knowledge, we provide the first study which investigates Wi-Fi fingerprinting-based indoor localization systems by considering room-level accuracy.

We have made signal strength (RSSI) measurements in rooms of different sizes and even behind the walls separating adjacent rooms; to discover the permeability of the walls and their effect on regenerating the borders of the rooms, by solely using the obtained RSSI values. For this purpose, we have only used raw measurement values, but not considered filters or estimation schemes of any kind. The rest of the paper is organized as follows. Section 2 reviews related works, Section 3 explains our setup, Section 4 provides experimental evaluations, Section 5 gives discussions and finally Section 6 includes the conclusions.

\section{Related Works}

Accuracy of indoor positioning systems are already subject to many recent researches. However, many of these studies focus solely on trilateration, which is hard to use in our scenario. That is because, when there are several rooms, and naturally many blocking walls, it is usually hard to produce a reliable distance estimation algorithm for the environment. The ones focus on fingerprinting, on the other hand, rarely analyze roomlevel localization cases.

$\mathrm{Li}$ et al. have studied on innovative ways of the fingerprinting approach (including deterministic and probabilistic methods), yet they claim trilateration is less attractive in office-style indoor places, where signal attenuation and fluctuation occurs due to high number of obstacles [3]. However, in their experiments, what they want to achieve (or test) is the absolute distance of the mobile unit relative to the APs. Likewise, their definition of success is the distance with the minimum error in terms of meters. However, rather we focus on determining the room; the mobile unit is located in.
In their experimental work, Seco et al. have done a relevant study, but using RFID tags and readers [4]. They have placed 71 passive RFID tags quasi-evenly placed in different rooms on a floor of an office building. Though there are too many rooms and obstacles in the $1600 \mathrm{~m}^{2}$ floor area, they have reached an accuracy of approximately 1.5 meters. But, they did not concern about the room-level accuracy, either. In addition, the mobile unit they have used, a large active RFID reader device, is impractical for everyday use in commercial and industrial applications, when compared to $\mathrm{Wi}-\mathrm{Fi}$ and Bluetooth modules, which are smaller and can be found in personal devices like mobile phones.

Mazuelas et al. developed another novel Wi-Fi-based IPS algorithm, and tested it using their existing infrastructure [5]. Nevertheless, they have considered trilateration approach and assumed existence of solid path loss/propagation models. That assumption is weak in terms of generalization. Because, different areas in any office environment may have different propagation characteristics, because of the distribution of the halls, walls and furniture (and of course, the crowd).

Jekabsons et al. worked on a Wi-Fi fingerprinting solution, which is completely based on the mobile device via a software developed by themselves [6]. They used the weighted average of inverse of RSSI distances, hence reached 2 to $2.5 \mathrm{~m}$ localization accuracy in their experiments. Even though their solution only requires the involvement of the mobile station and is considerably accurate; for every subject place, a map of the place should be installed to the software and the training phase should be done over this map, which is not so practical.

Researchers in Aruba Networks, a company based in the US, made a very comprehensive experimental study and documented it as a white paper [7]. Their work consists of detailed attenuation and coverage statistics of $\mathrm{Wi}-\mathrm{Fi}$ signals as well as visual heat maps and track paths in an office environment. The 70-page study is clearly a good guide for $\mathrm{Wi}-\mathrm{Fi}$ fingerprint based indoor positioning applications. Nevertheless, does not conclude anything related to the room-level positioning accuracy.

Besides all of the above works, there is an Android application available on the Play Store, called Room+ [8]. It is based on a brand-new open source project: The Framework for Internal Navigation and Discovery (FIND). This easy-to-use program allows saving fingerprints of places via a training phase and allows user to name the place, where a fingerprint is created. According to our trials, regardless of the number of the places (fingerprints), as long as there are at least a $2 \mathrm{~m}$ distance between them, the program is somewhat successful on determining the current place of the host (mobile) device. However, the program is designed to force itself to choose the best matching place per to prerecorded fingerprints database. This also happens when the mobile device is in a completely irrelevant and/or far 
away location. Hence, despite being a stable application, it may be misleading in our case.

\section{Materials and Methods}

This section introduces the rationale behind this study, and the required infrastructure, as well as the test cases. Table 1 summarizes the main assets of our setup.

\subsection{Use Case Scenario}

In a broader research, being conducted in Ege University in Izmir; researchers work on tracking activities in particular rooms of the university hospital, in which patients with critical health conditions stay, in order to ensure their safety and protection. Since these patients' immune system usually becomes very weak, even doctors and other healthcare personnel should be very careful when approaching them. However, it is always under question by the hospital management and patient companions that if they really pay enough attention, as literature points out, too $[9,10]$. Hence, the rationale behind this study emerges, as with the question: "How well we can detect if a person-of-interest (a doctor in that case) approaches and enters a room (a patient's room, likewise)?"

From here, we have demonstrated the given scenario with two cases: detection at the anchor station and detection at the mobile unit. First one implies a fixed station located in the room detects the mobile station. In the second, the fixed station stays passive and mobile station detects whether it is in a room or not. Additionally, we also examined the effect of walls on radio signal attenuation.

\subsection{Devices}

An Android based OnePlus One smartphone equipped with a Qualcomm WCN3680 Wi-Fi module and a Skyworks SKY85709-11 WLAN front-end module is used as the receiver mobile station to track surrounding APs and measure the RSSI values. ZTE Axon 7 Mini, another Android based smartphone equipped with a Qualcomm X12 LTE/Wi-Fi modem, is used as the fixed station in the Wi-Fi hotspot mode.

\subsection{Test Environment}

The experimental study that consists of $\mathrm{Wi}-\mathrm{Fi}$ radio signal tracking and measurement was held in the block $\mathrm{C} 1$ in the main building of engineering faculty in Yalnizbag Campus of Erzincan University. The building was made of precast and reinforced concrete, while the internal walls those separate the rooms are aerated concrete of $15 \mathrm{~cm}$ width. Additionally, the doors (shown as dotted lines in the figures) are made of wooden chipboard with a width of $5 \mathrm{~cm} .12$ Enterasys RBT-412 $\mathrm{Wi}-\mathrm{Fi}$ router/modems were already installed onto the building walls and actively serving Wi-Fi connectivity for the Internet. They may (or may not) cause nonnegligible attenuative noise and/or fading. Therefore, it is worthwhile to state as a side note.

\subsection{Software}

An Android application with Wi-Fi listing feature is developed and installed in the above-mentioned mobile device. Apart from listing the all discovered Wi-Fi broadcasting devices, the program also allows the user to set an RSSI-based alarm threshold for each sensed Wi-Fi broadcasting device. Hence, it is possible to get notified whenever a station approaches to a close proximity (determined by the threshold value) or leaves. The same applies to conditions, where the APs are fixed but the mobile station is approaching to them, and vice versa. This feature itself will be enough to determine if a user is in the control room or not; as long as our hypothesis is verified during the experimental studies.

\section{Experimental Study}

The experiments we have conducted are presented in this section with supporting graphical material. It is highly recommended for the reader to refer to the color version of this paper. Those common parameters apply for all tests mentioned in the rest of the paper: Tests were made during the daytime between 09:00 am and 17:00 pm; when the indoor temperature was between 22 and $28 \mathrm{C}$. There were no people in the rooms during the tests, except for one, who held the mobile device. The AP and the mobile station were held always at a height of $1.5 \mathrm{~m}$. We also state that none of the rooms were containing any obstacle (i.e. furniture) taller than $1 \mathrm{~m}$. Last but not least, all RSSI measurements for every cell and in every room, are repeated 5 times after a waiting period of 1 minute between each attempt. Yet, mean average of these measurements are given in all of the figures below. Nevertheless, in none of these measurement sets of 5, extreme values exceed the mean average by $\% 10$. The color-legend is as follows for all figures below, except for Figure 5 and 6; red for -30 to $-39 \mathrm{dBm}$, orange for 40 to $-49 \mathrm{dBm}$, yellow for -50 to $-59 \mathrm{dBm}$ green for -60 to $-69 \mathrm{dBm}$, turquoise for -70 to $-79 \mathrm{dBm}$, purple for -80

Table 1. Summary of the assets and settings of our setup.

\begin{tabular}{||c|c|c|c||c|c||c||}
\hline \multicolumn{5}{|c||}{ Environment } & \multicolumn{1}{c||}{ Hardware } & Software \\
\hline Type & Size $\left(\boldsymbol{m}^{\mathbf{2}}\right)$ & Nr. of Cells & Qty & Type & Features \\
\hline $\begin{array}{c}\text { Small } \\
\text { Room }\end{array}$ & $3 \times 3$ & 3 & 3 & $\begin{array}{c}\text { Mobile } \\
\text { Station }\end{array}$ & $\begin{array}{c}\text { OnePlus One; with Qualcomm } \\
\text { WCN3680 \& Skyworks SKY85709 }\end{array}$ & Makes periodic Wi-Fi scans \\
\hline $\begin{array}{c}\text { Medium } \\
\text { Room }\end{array}$ & $3 \times 5$ & 12 & 3 & $\begin{array}{c}\text { Access } \\
\text { Point }\end{array}$ & $\begin{array}{c}\text { ZTE Axon 7 Mini; with X12 } \\
\text { LTE/Wi-Fi }\end{array}$ & $\begin{array}{c}\text { Lists all existing SSIDs, } \\
\text { manually assign RSSI thresholds }\end{array}$ \\
\hline $\begin{array}{c}\text { Large } \\
\text { Room }\end{array}$ & $\begin{array}{c}11.5 \times 9.5 \text { and } \\
9.5 \times 9.5\end{array}$ & 24 and 20 & 2 & $\begin{array}{c}\text { Noise } \\
\text { Sources }\end{array}$ & $12 \times$ Enterasys RBT-412 AP & $\begin{array}{c}\text { Keeps track of RSSI values for } \\
\text { selected APs }\end{array}$ \\
\hline \hline
\end{tabular}


to $-89 \mathrm{dBm}$ and magenta for -90 to $-99 \mathrm{dBm}$.

\begin{tabular}{|c|c|c|c|c|c|c|c|c|}
\hline \multicolumn{3}{|c|}{ Room M1 } & \multicolumn{3}{c|}{ Room M2 } & \multicolumn{3}{c|}{ Room M3 } \\
\hline-58 & -54 & -47 & -35 & -34 & -34 & -48 & -55 & -60 \\
\hline-58 & -55 & -51 & -44 & -40 & -41 & -52 & -55 & -58 \\
\hline-57 & -57 & -53 & -47 & -43 & -42 & -55 & -58 & -57 \\
\hline-58 & -58 & -53 & -49 & -49 & -52 & -53 & -57 & -57 \\
\hline-60 & -57 & -55 & -55 & -54 & -53 & -54 & -62 & -64 \\
\hline-61 & -55 & -54 & -57 & -56 & -52 & -53 & -62 & -64 \\
\hline
\end{tabular}

Figure 1. Measurements in medium-sized rooms (dBm).

Figure 1 above illustrates a heatmap per to RSSI values regarding the Wi-Fi signals emitted by a fixed active access point, located in the marked cell, on the northmost wall of the center room, namely M2. Yet there are 3 adjacent rectangular rooms included in the experiment space, all of which are divided into equal cells as a grid. The room size is $3 \times 5$ sq. meters, for all rooms; while the cell size is $1 \times 1.25$ sq. meter. For comparison, when the mobile station is placed just on the fixed station, so that they touch each other, the RSSI is measured as $-20 \mathrm{dBm}$. Yet, all measurements are made in the center of each cell. So that the measurement in the cell which contains the fixed station is made $50 \mathrm{~cm}$ away from it.

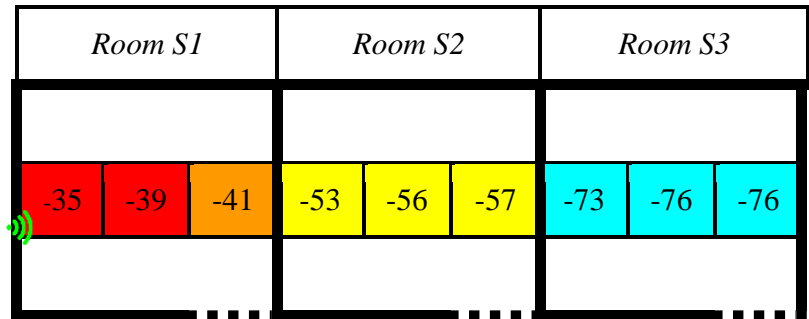

Figure 2. Heatmap showing radio attenuation on a linear track containing 2 walls in small rooms $(\mathrm{dBm})$.

Figure 2 reflects the attenuation effects of consecutive internal walls on the power of the observed radio signal. Here, a linear cell sequence is formed, which includes spaces in three neighboring room (same rooms as the above). The rest of the room areas (marked as whitespace) are ignored. The cell size is 1 x 1 sq. meter, while the room width is $3 \mathrm{~m}$. The AP is placed on the leftmost wall in Room S1 and the doors were kept closed.

Like Figure 2; Figure 3, gives an inter-room attenuation pattern. But now, the fixed station is mounted on the wall, which separates two rooms of interest, rather the farthest edge in the Room S1. The doors were again kept closed. The test with medium sized rooms, illustrated in Figure 1 , is repeated in larger rooms with minor differences in the focus. In this step of the experiment, the main purpose is to check the attenuative effect of the walls, in the case of the first wall that the radio signals encounter is farther away. So, bigger rooms with farther walls are considered.

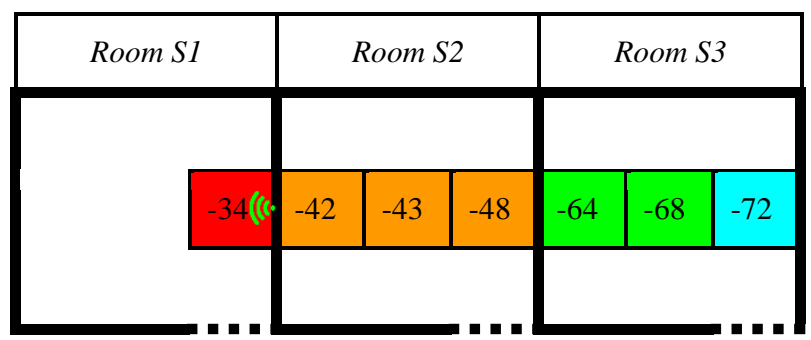

Figure 3. Heatmap showing radio attenuation on a linear track containing 2 walls in small rooms, where the AP is placed on a common wall $(\mathrm{dBm})$.

Moreover, the AP is now placed in the room L1, on the furthest wall from the neighboring room (L2). The room sizes are $11.5 \times 9.5$ sq. $\mathrm{m}$. and $9.5 \times 9.5$ sq. m. for rooms L1 and L2, respectively. The cell size is $2.4 \times 1.9$ sq. m. for both rooms. The results are presented in the Figure 4.

\section{Validation and Discussion}

By the obtained results given in the previous section, we have found that, it is possible to consider an approximate RSSI threshold for any (geographically plain, i.e. rectangular) room made of solid walls, so that the roomlevel positioning can feasibly be done via only one AP. In the example test scenario we have conducted, for the room M2, where the test AP is located, shown in the Figure 1, an RSSI localization threshold of -50 to -52 $\mathrm{dBm}$ (depending on the required sensitivity) shall be significant, since most of the cells in the room has a smaller RSSI fingerprint, while most of the area outside has a larger RSSI fingerprint value. As shown in Figure 5 , we have changed the color-legend of the rooms previously given in Figure 1, such that, the cells with an RSSI value smaller than $-50 \mathrm{dBm}$ are marked as red (representing the area of the room-of-interest), while the ones with larger RSSI are marked as green (representing the outer irrelevant areas).

Likewise, the same trick was applied to the scenario containing larger rooms given in the Figure 4. Here, we considered a threshold of $-65 \mathrm{dBm}$ and changed the color-legend accordingly. In Figure 6, cells with an RSSI smaller than $-65 \mathrm{dBm}$ are marked as red, while the ones with larger RSSI are marked as green. As can clearly be seen in both Figures 5 and 6; theoretically it is very easy to determine if (a person with) a mobile station is whether in the room-of-interest or not, with a considerably small error rate, on the mobile station side. By the way, we have selected our threshold values to favor allowing false positives over false negatives. Which means, if a prospective application based on this method detects inclusion of the mobile device into the room, it can actually be outside, too (failed detection). But, when the application claims that the mobile device is outside, it should highly probable be outside. These RSSI thresholds could however be selected as vice versa. 


\begin{tabular}{|c|c|c|c|c|c|c|c|c|c|c|c|}
\hline \multicolumn{5}{|c|}{ Room L1 } & \multicolumn{5}{c|}{ Room L2 } & \\
\hline-54 & -54 & -56 & -58 & -61 & -58 & -67 & -70 & -73 & -75 & -76 & -90 \\
\hline-37 & -44 & -54 & -56 & -57 & -64 & -64 & -68 & -71 & -76 & -77 & -86 \\
\hline-9$))-38$ & -46 & -55 & -56 & -58 & -61 & -72 & -72 & -73 & -74 & -77 & -84 \\
\hline-56 & -52 & -54 & -57 & -60 & -62 & -71 & -74 & -80 & -77 & -75 & -84 \\
\hline-64 & -70 & -66 & -68 & -68 & -72 & -69 & -70 & -68 & -74 & -76 & -82 \\
\hline
\end{tabular}

Figure 4. Measurements in larger rooms $(\mathrm{dBm})$.

\begin{tabular}{|c|c|c|c|c|c|c|c|c|}
\hline \multicolumn{3}{|c|}{ Room M1 } & \multicolumn{3}{c|}{ Room M2 } & \multicolumn{3}{c|}{ Room M3 } \\
\hline-58 & -54 & -47 & -35 & -34 & -34 & -48 & -55 & -60 \\
\hline-58 & -55 & -51 & -44 & -40 & -41 & -52 & -55 & -58 \\
\hline-57 & -57 & -53 & -47 & -43 & -42 & -55 & -58 & -57 \\
\hline-58 & -58 & -53 & -49 & -49 & -52 & -53 & -57 & -57 \\
\hline-60 & -57 & -55 & -55 & -54 & -53 & -54 & -62 & -64 \\
\hline-61 & -55 & -54 & -57 & -56 & -52 & -53 & -62 & -64 \\
\hline
\end{tabular}

Figure 5. Re-painted color-legend per to threshold values for the room M2, previously shown in Figure $1(\mathrm{dBm})$.

In our scenarios, the detection was made by the mobile device itself, which may not be so useful in some commercial/industrial applications. However, that is not a noteworthy issue; since it is very easy to modify the mobile software, so that the mobile device announces its detections to a web/application server or surrounding devices (i.e. APs), if they also have similar software.

The main problem is the unbalanced and unreasonable instant fluctuations of the RSSI values. The strength of radio signals usually (almost constantly) change over time, on the receiver side. These changes are caused by many observable and unobservable environment conditions. Mostly, they do not affect the quality of given services like the internet connection and can be neglected. But, for our methodology, it will significantly reduce the effectiveness and accuracy. These fluctuations we have experienced, were surely not surprising since this topic has been studied by many researchers recently [11]. However, as stated previously, we have an important statistical information that can be exploited to increase the practical accuracy of the proposed method. It is that, none of the measured RSSI values for any cell (remind we have measured 5 RSSIs for each cell), exceed the mean average by $\% 10$. So, $\% 10$ can be assumed as a worst-case variance for all cells, and we can then calculate a backed-up room threshold by considering this. In the light of this information, use of smart filtering and/or estimation algorithms, like Kalman Filter, (which are not studied in this work) will surely provide a huge increase on accuracy of room-level positioning systems.

From the cost point of view; this approach loses its feasibility, if all or most rooms should be tracked. Because, in this case, all or most rooms should be equipped with a Wi-Fi access point, which would obviously not be cheap. However, the cost is still controversial since the number of people to be served is theoretically unlimited. That's because no mobile device shall need to connect these access points, they only need to listen (sniff) the existing radio signals. On the other hand, if there are only several "important" rooms to be tracked, then this single AP approach requires a very small investment and is very cost-efficient.

\section{Conclusion}

Many previous studies, as mentioned earlier, focus on estimating absolute positions of the objects, using means of coordinate planes on relative locations, like building maps and floor plans. Nevertheless, locationing does not enforce absolute positioning, so that relative positions can also be used in these relative locations. A trademark of our work is the use of room notion as a matter of relative locationing. In this work, we have shown that it is possible to estimate the room-wise location of an object, considering an existing $\mathrm{Wi}-\mathrm{Fi}$ infrastructure, solid concrete walls and some other configurations. While the presented estimates are only valid in certain environment conditions, and still pose some false positive or false negative detections, we were able to disclose roomspecific RSSI heatmaps, for every single room that has a dedicated wireless access point. The capability of finding out the room, in which an object or person is currently located may unveil new opportunities for security, automation and logistics applications, apart from the problem we have originally focused on in this project. 


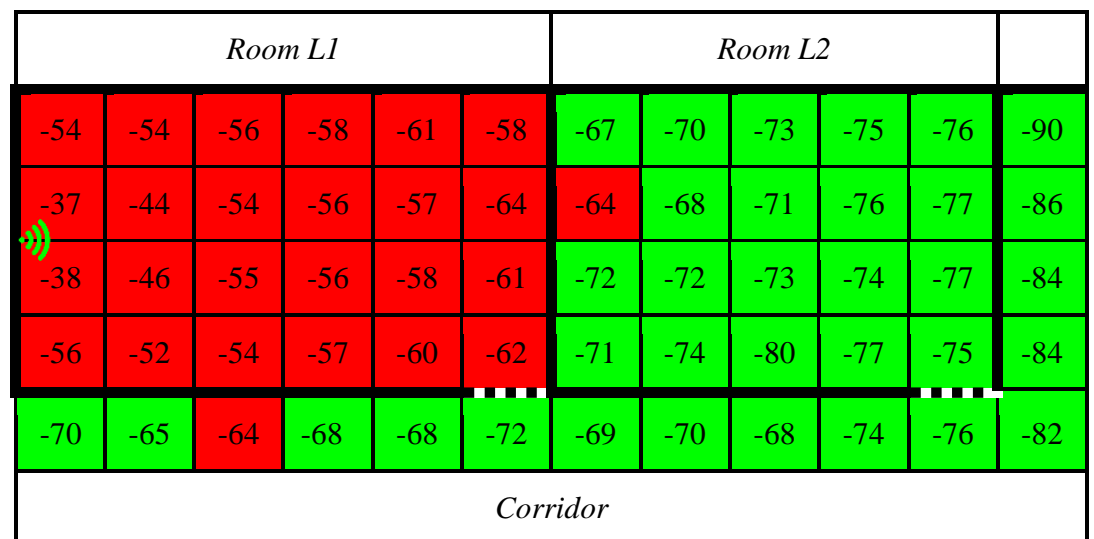

Figure 6. Re-painted color-legend per to threshold values for the room L1, previously shown in Figure 4 (dBm).

Besides all these advantages, there are three major issues on such an application scenario. First, in case of large halls, lounges and classrooms, bigger than 100 sq. meter (e.g. $10 \times 10$ ), the attenuation patterns may be different and be less significant, because of the potentially reduced correlation between the RSSI and obstructions due to longer distances. Second, the power consumption on the mobile devices (carried by the persons or objects) will presumably be high, since they should be in their active mode, all the time. And third, RSSI fingerprints recorded in a building may be subject to change eventually, due to seasonal temperature changes, temporary populousness, setup or removal of electronic devices etc. In fact, they may even change slightly from day to night. Considering these critiques, as a future work, we will study the correlation between the room size (distance to the walls) and the attenuative effect of the walls, in addition to the effects of the seasonal conditions. So that, we will be able to derive a formula in order to consider a decision threshold without making custom learning tests to create fingerprints, which will lead to automatize the process.

\section{Acknowledgments}

We want to thank Abdullah Cavdar, researcher in Erzincan University, for the equipment he provided. Orhan Dağdeviren acknowledges the research project 2016/UBE/002, which is funded by Ege University.

\section{Authors' Contributions}

Umut Can Çabuk: Drafted and wrote the manuscript, designated the setup and performed the experiments.

Feriştah Dalkılıç: Worked on data visualization. Assisted the analytical analysis of the setup and results.

Orhan Dağdeviren: Provided theoretical background. Supervised the experiment's progress, interpreted the results and did proofreading.

\section{Ethics}

There are no ethical issues after the publication of this manuscript.

\section{References}

1. Kim, S, Ha, S, Saad, A, Kim, J. Indoor Positioning System Techniques and Security, in proc. of the IEEE Fourth International
Conference on e-Technologies and Networks for Development (ICeND), Lodz, Poland, 2015, pp 1-4.

2. Koyuncu, H, Yang, S.H, A survey of indoor positioning and object locating system, International Journal of Computer Science and Network Security, 2010, 10(5), 121-128.

3. Li, B, Salter, J, Dempster, A.G, Rizos, C. Indoor Positioning Techniques Based on Wireless LAN, proceedings of the IEEE International Conference on Wireless Broadband and UltraWideband Communications (AusWireless), Sydney, Australia, 2006.

4. Seco, F, Plagemann, C, Jiménez, A.R, Burgard, W. Improving RFID-Based Indoor Positioning Accuracy Using Gaussian Processes, proceedings of the IEEE Int. Conference on Indoor Positioning and Indoor Navigation (IPIN), Zurich, Switzerland, 2010, pp 1-8

5. Mazuelas, S, Bahillo, A, Lorenzo, R.M, Fernandez, P, Lago, F.A, Garcia, E, Blas, J, Abril, E.J, Robust indoor positioning provided by real-time RSSI values in unmodified WLAN networks, IEEE Journal of selected topics in signal processing, 2009, 3(5), 821831. doi:10.1109/JSTSP.2009.2029191.

6. Jekabsons, G, Kairish, V, Zuravlyov, V, An analysis of Wi-Fi based indoor positioning accuracy, Scientific Journal of Riga Technical University, Computer Sciences, 2011, 44(1), 131-137. doi:10.2478/v10143-011-0031-4.

7. Aruba Networks Inc., Indoor $802.11 \mathrm{n}$ site survey and planning. https://community.arubanetworks.com/aruba/attachments/aruba/u nified-wired-wireless-access/588/1/indoor80211n_2012-0531.pdf, 2012 (accessed 12.04.2018)

8. Internal Positioning, Framework for internal navigation and discovery (FIND). https://www.internalpositioning.com/faq/, 2017 (accessed 12.04.2018)

9. Group, H.L, Hand washing: A modest measure-with big effects, BMJ: British Medical Journal, 1999, 318(7185), 686.

10. Whitby, M, McLaws, M. L, Ross, M.W, Why healthcare workers don't wash their hands: a behavioral explanation, Infection Control \& Hospital Epidemiology, 2006, 27(5), 484-492. doi:10.1086/503335.

11. Chapre, Y, Ignjatovic, A, Seneviratne, A, Jha, S. Csi-mimo: Indoor Wi-Fi Fingerprinting System., in proc. of the IEEE $39^{\text {th }}$ Conf. on Local Comp. Networks (LCN), Edmonton, AB, Canada, 2014, pp 202-209. 